\title{
Población venezolana en el contexto de la crisis sanitaria en Lima Metropolitana
}

Sección ESTUDI0S

RECIBIDO: 01/07/2021

APROBADO: $15 / 08 / 2021$

PUBLICADO ONLINE: 30/11/2021

\author{
Rubén Ticona Fernández Dávila \\ Universidad Ricardo Palma \\ rubenticonafd@gmail.com.pe \\ https://orcid.org/0000-0002-4601-4542
}

\section{RESUMEN}

El presente artículo aborda la temática de la migración y la crisis sanitaria. Tiene como objetivo identificar las políticas públicas adoptadas por el Perú frente a la situación provocada por la pandemia de la covID-19, se analiza desde la perspectiva de población y desarrollo, los impactos de la crisis sanitaria en grupos de población en situación de mayor vulnerabilidad como son las personas migrantes. Sostenemos que la población migrante venezolana ha quedado en una situación de indefensión frente a la crisis sanitaria. Metodológicamente el artículo plantea un análisis mixto, combina análisis de información estadística, así como análisis de discurso y entrevistas a profundidad. Finalmente, se afirma la necesidad de conocer y reconocer la problemática que atraviesan los migrantes en el país para mayor eficiencia y eficacia del Estado peruano en el despliegue de sus políticas públicas.

PALABRAS CLAVE: Derechos humano, población migrante, políticas públicas, crisis sanitaria, Estado.

\section{Human rights of the Venezuelan migrant population in the context of the health crisis in Metropolitan Lima}

\section{ABSTRACT}

This article addresses the issue of migration and the health crisis. Its objective is to identify the public policies adopted by Peru in response to the situation caused by the COVID-19 pandemic; it is analyzed from the perspective of population and development, the impact of the health crisis on the most vulnerable population groups, such as migrants. We maintain that the Venezuelan migrant population has been left in a situation of defenselessness in the face of the health crisis. Methodologically the article proposes a mixed analysis, combines analysis of statistical information, as well as discourse analysis and in-depth interviews. Finally, it affirms the need to know and recognize the problems faced by migrants in the country to increase the efficiency and effectiveness of the Peruvian State in the deployment of its public policies.

KEYWORDS: Human rights, migrant population, public policies, health crisis, state.

(c) Los autores. Este artículo es publicado por la Revista de Sociología de la Facultad de Ciencias Sociales, Universidad Nacional Mayor de San Marcos. Este es un artículo de acceso abierto, distribuido bajo los términos de la licencia Creative Commons Atribución 4.0 Internacional (CC BY 4.0) [https://creativecommons.org/licenses/by/4.0/deed.es] que permite el uso, distribución y reproducción en cualquier medio, siempre que la obra original sea debidamente citada de su fuente original. 


\section{Introducción}

$\mathrm{P}$

erú actualmente se encuentra atravesando por la mayor crisis sanitaria que le ha tocado afrontar debido a la propagación de la covid -19. No obstante, esta crisis sanitaria si bien está ligada a la pandemia, su origen se encuentra más allá de este. Es decir, el origen de esta gran crisis no ha sido otra que el Estado peruano y los gobiernos de turno que han pasado por este. La nula capacidad de gestión, los altos niveles de corrupción, obras incompletas, un sistema de salud colapsado y un sistema educativo en declive es la herencia que nos han dejado cinco presidentes que ahora están siendo investigados por corrupción. La covid -19 no ha hecho más que evidenciar esto y acrecentar las brechas socioeconómicas existentes, en un país donde el acceso a un sistema de salud y a un sistema educativo de calidad es un privilegio para quien tiene los recursos suficientes.

Esta pandemia según los reportes oficiales hasta el 26 de abril había un aproximado de 60 mil muertes hasta la fecha según las cifras oficiales publicadas por el MINSA; mientras que el SINADEF señala que el Perú supera las 153 mil muertes por la COVID-19, según las cifras actualizadas por el Estado peruano a la fecha hay $198 \mathrm{mil}$ muertes causadas por el CovID-19. Sin embargo, el $80 \%$ de estas pertenecen a los sectores poblacionales de mayor vulnerabilidad. Entonces ¿de quién es el rostro del fallecido? Del informal, del marginado, del pobre, del desplazado y del migrante. Es en este panorama que surge la necesidad de reflexionar sobre las poblaciones que reúnen todas estas características siendo una de estas la población venezolana en el Perú, entre el olvido y el silencio, entre la informalidad y la pobreza. La condición del migrante venezolano viene siendo invisibilizada por los medios de comunicación ni por el aparato estatal, dando la apariencia de la no existencia de este fenómeno.

La población migrante venezolana en el Perú ha pasado por diversos momentos de transición, por lo cual, tomaremos como referencias los años del 2016 al 2021, de esta manera podremos ver la evolución histórica de la población venezolana en el Perú; tomando como referencia al proceso migratorio la crisis política, económica y social por la que atraviesa Venezuela con el gobierno de Nicolás Maduro. Como señala Koechlin, Vega \& Solórzano (2018):

Hugo Chávez se encontraba como presidente de la República y fue reelegido por tres períodos consecutivos hasta su fallecimiento el 5 de marzo de 2013. Desde entonces, el gobierno fue asumido por su vicepresidente y luego elegido presidente de la República, Nicolás Maduro. Durante todo ese período, los partidos opuestos al régimen han realizado presiones y denuncias al Gobierno, y han tomado diversas acciones de protesta. Esta tensión política interna se visibilizó en el escenario 
mundial por la solicitud de presencia de observadores y organismos internacionales para intervenir en temas relativos a los derechos humanos y a las libertades fundamentales. Es así como la política interna venezolana cobró visibilidad en la política internacional; sobre todo, a nivel regional, por el apoyo de países cuyos Gobiernos simpatizaban con el régimen bolivariano, como Brasil, Argentina y Bolivia, y obtuvo la crítica de países como Colombia y Chile y, posteriormente, el Perú, ubicados en las antípodas políticas y económicas del régimen bolivariano (p. 54).

Todo ello ha ocasionado que millones de venezolanos decidiesen migrar a otros países de la región debido a la crisis política, social y económica que atravesaba su país por el gobierno de Nicolás Maduro. Ante esta situación el entonces presidente Pedro Pablo Kuczynski decidió crear al grupo de Lima, una organización política internacional que tenía como finalidad derrocar al régimen chavista y evitar la catástrofe social.

Según las cifras oficiales de migraciones y del Banco Mundial, en el año 2016 la población venezolana en el Perú era de unos aproximados 27 mil personas. Para el año del 2017, con el discurso del entonces presidente Pedro Pablo Kuczynski en la OEA y la apertura de las fronteras, esta cifra crecería exponencialmente a unas 200 mil personas. En el año de 2018, la población venezolana en el Perú bordearía las 635 mil personas aproximadamente. Mientras que, en el 2019, esta cifra crecería a 862 mil personas. El 2020, marcado por la propagación de la COVID-19, el inicio de la cuarentena y la crisis sanitaria, la población venezolana aumentó a un 1 millón 43 mil personas aproximadas. Cifra que a inicios del 2021 crecería a 1 millón 200 mil personas. Constituyéndose como la mayor población migrante en el Perú. No obstante, la población con menos derechos, la de mayor vulnerabilidad y la que se encuentra en la informalidad y la pobreza. Considerando lo mencionado en párrafos anteriores, si el sistema de salud, laboral y educativo ya se encontraba colapsado para los mismos peruanos, razón por la cual, la población venezolana ha quedado relegada al acceso a los servicios básicos que plantean los derechos humanos y la agenda 2030.

Según un informe presentado por Panorama en el 2020 se señala que "en cada esquina vemos ambulantes venezolanos, las mujeres venezolanas son víctimas de la trata de personas y prostitución mientras que los niños venezolanos no pueden acceder al derecho de tener una educación básica de calidad, muchos de ellos deben de trabajar para poder ganarse el sustento de cada día". Por el motivo, que muchos de los migrantes venezolanos no llegaban a un mercado formal de trabajo; sino todo lo contrario, llegaba a un sector informal de trabajo marcado por la precarización laboral y la falta de derechos. 
Por ello, el presente artículo tiene como finalidad abordar la condición de la población venezolana en el Perú durante la crisis sanitaria de la Covid-19 y las consecuencias que este ha originado en ella; enfatizando en que esta población ha quedado en vulnerabilidad frente al acceso de derechos, así como, la ausencia de políticas públicas y de una acción estatal por atender esta problemática.

La parte cualitativa de este trabajo de investigación incluyó una etnografía digital, una revisión sistemática de los discursos y las representaciones imaginarias sobre la población migrante venezolana en el Perú. Para tal motivo, se realizó un análisis documental sobre un total de 245 noticias publicadas en la página web de El Comercio publicadas durante los años del 2016 al 2021 que tenían como temática a la población venezolana en el Perú.

En un primer apartado, nos centraremos en analizar la situación económica y laboral de la población migrante venezolana. Recordemos que, desde hace unos 10 años, en el Perú las tasas de desempleo han ido creciendo exponencialmente, a ello, ahora sumemos la inserción de aproximadamente 1 millón de personas. Pero, nuestra adición no queda allí, ahora sumémosle el incremento del desempleo que ha ocasionado la pandemia de la covid-19. Esta situación ha conllevado a dos consecuencias directas: i) la población venezolana ha quedado relegada al sector informal; ii) el proceso de estigmatización a la población venezolana, frases tales como: "este venezolano me está quitando el empleo" no son más que el inicio de un proceso de xenofobia marcado en la sociedad peruana.

En un segundo apartado, nos centraremos en analizar este proceso de estigmatización de la población venezolana, lo cual, ha originado una fuerte xenofobia en gran parte de la población peruana. Motivo por el cual, analizaremos las noticas de unos de los diarios más importantes del Perú, elaborando un diagnostico por año y la evolución de las noticias en torno a la población venezolana en el Perú.

En un tercer apartado, nos centraremos en analizar a una población venezolana en vulnerabilidad, estigmatizada, sin trabajo frente a una crisis sanitaria y la ausencia del Estado. Revelando que, esta población es la que menos derechos tiene y es la que sufre más las consecuencias de la desigualdad del Perú. Finalmente nos plantemos la necesidad de abrir el debate en este tema que hasta la fecha no ha sido tocado.

Por último, presentaremos las conclusiones, donde reflexionaremos acerca del migrante venezolano como la población más indefensa frente a la crisis sanitaria que está atravesando el país. Asimismo, señalaremos que las políticas públicas desde el Estado han sido ineficientes e inexistentes frente al éxodo venezolano en el país. Una población sin derechos ni accesos a los servicios básicos que es invisibilizada por los medios de comunicación y por las estadísticas nacionales. No 
solo mueren peruanos en esta pandemia, también venezolanos, por tal motivo nos proponemos visibilizar ello.

\section{De lo formal a lo informal: situación laboral de la población venezolana en el Perú}

Desde la llegada de la población venezolana al Perú, el gran problema que surgía se dio en la inserción de esta a la PEA. Según las cifras del Ministerio del Trabajo en su publicación "Políticas Nacionales de Empleo" (2012), sostiene que se deberían de generar alrededor de 250 mil empleos para lograr contrarrestar las tasas de desempleo (p. 27); sin embargo, con la llegada de la población venezolana estas cifras se han incrementado considerablemente y ha agravado las condiciones del sector formal de trabajo. De estas cifras, hemos de mencionar que solo un $30 \%$ es cubierto por el sector formal de trabajo mientras que el $70 \%$ recae en el sector informal; así como lo detalla Tenorio (2020) en su investigación titulada“El empleo informal en el Perú: Una breve caracterización 2007-2018".

Según las últimas cifras presentadas por el Banco Mundial (2020) en su artículo: "Infografía: Migrantes y Refugiados Venezolanos en El Perú: El Impacto de la Crisis de la Covid-19 (\#Coronavirus)", detalla que solo el 6\% de la población venezolana se encuentra en el sector formal. Por otro lado, el $67 \%$, se encuentra en el sector informal. De los cuales, si desagregamos las cifras oficiales, un gran porcentaje de esta población se encuentra entre la explotación y la precariedad laboral.

Dávalos (2020) menciona que "los migrantes y refugiados venezolanos se encuentran en empleos más vulnerables debido a la alta informalidad y su concentración en pequeñas empresas" (p. 1). Existen tres factores claves por los cuales estas empresas han decidido contratar a la población venezolana en lugar que a la población peruana: i) salarios inferiores al salario mínimo; ii) la no responsabilidad jurídica como empleadores; iii) el no reconocimiento de derechos laborales.

Para Rojas \& Monterroso (2019) “la diáspora venezolana, que puede ser considerada como cualificada, no está siendo efectivamente aprovechada por el mercado laboral peruano, por cuanto está desaprovechando individuos de alta calificación en empleos informales y de bajo desempeño a nivel estratégico" (p. 144).

Una encuesta realizada por Condori et al. (2020) a 249 venezolanos en la ciudad de Huancayo, muestra los siguientes resultados que no solo hacen más que confirmar lo que veníamos anunciando en párrafos anteriores: 
El 100\% de los/as encuestados/as asevera estar en condición de subempleado/a. Efectivamente, el $59.8 \%$ es trabajador/a dependiente y el $40.2 \%$ trabaja por su propia cuenta. De los/as trabajadores/as dependientes: el 53\% trabaja sin contrato, el 35.6\% está contratado/a temporalmente y el $11.4 \%$ tiene contrato indefinido. Respecto a la jornada laboral: el 53.8\% trabaja entre 9 a 12 horas diarias, 21.3\% más de 12 horas, sólo un $10.8 \%$ trabaja 8 horas y $14.1 \%$ labora menos de 8 horas diarias. El ingreso mensual que percibe el mayor porcentaje (74.3\%) es inferior al ingreso mínimo vital (p. 78).

Los resultados mostrados por Condori et al. (2020), no solo se repiten en otras regiones del Perú, sino que se agudizan. Un mercado que desborda por el exceso de mano de obra ha llevado a muchos venezolanos a trabajar de manera ambulatoria por diversas calles del Perú, a aceptar trabajos informales sin un contrato ni derechos laborales (salario mínimo, gratificaciones, vacaciones anuales, CTS, seguro de salud, entre otros) y a trabajar por jornadas mayores a las 8 horas en condiciones de sobreexplotación y precariedad. De igual manera, el mismo trabajo en mención señala lo siguiente:

El INEI (2019) encontró que, el 91.5\% de venezolanos en el Perú viene desarrollando alguna actividad económica, de éstos el $78.3 \%$ son empleados dependientes, y el $88.5 \%$ trabaja sin contrato laboral, la mayoría se insertó laboralmente en actividades económicas distintas a su formación académica, el 69.4\% trabajan de 51 a más horas semanales, obteniendo un ingreso mensual de 1116 soles que lo obtienen por su actividad económica principal y secundaria (como se citó en Condori et al, 2020, pp. 78-79).

Sin embargo, habría que mencionar que, para la fecha, estas cifras se han acentuado e incrementado debido a la pandemia y crisis sanitaria por la CovID-19. Además de ello, según la última encuesta realizada por EMPOVE 2018, la mayoría de la población venezolana trabajaba en los rubros de: hotelería, restaurantes, comercio, transporte y construcción, sectores donde la tasa de informalidad es mucho mayor que a otros. Estos rubros fueron los más afectados durante la pandemia, con decir que un total de 70 mil restaurantes han cerrado debido a la crisis sanitaria existente. Para la Cámara de Turismo del Perú (2021):

El panorama económico dentro de la nación no sería el mismo. Esto debido a que el desarrollo del sector de Alojamiento y Restaurantes se ha visto duramente comprometido por la pandemia de la COVID -19, registrando una pérdida económica del $50.45 \%$ en comparación con el año anterior. Asimismo, la Asociación Peruana de Hoteles, Restaurantes y Afines (Ahora Perú) revela que, de los 200.000 restaurantes 
registrados, 70.000 cerraron debido a la pandemia, lo cual tendría como resultado el desempleo de más de 350,000 ciudadanos.

Hecho que no solo incrementa el desempleo en el Perú, sino que agudiza y precariza la situación económica y laboral de la población venezolana. Según los últimos informes realizados por ACNUR en conjunto con el Banco Mundial y la ONU, aproximadamente un 18\% de la población venezolana en el Perú se encontraba en situación de pobreza y pobreza extrema en el año del 2020. Es muy probable que esta se haya incrementado considerablemente por las nuevas medidas de confinamiento adoptadas por el gobierno de Sagasti llevando a una situación de vulnerabilidad a la población venezolana en el Perú.

Otro aspecto relevante para mencionar es lo señalado por Rojas \& Monterroso (2019) sobre la población venezolana en el Perú: “La migración venezolana en el Perú tiene una connotación altamente económica; debido a que los inmigrantes, además de huir de la precaria situación que se vive en su país, tienen dentro de sus objetivos fundamentales insertarse en el mercado laboral peruano" (p. 142).

Evidenciando que la mayoría de la población venezolana en el Perú sigue viendo la forma de insertarse dentro del sistema laboral peruano sin importarle las condiciones del trabajo ni las exigencias. Ello ha conllevado a desarrollarse otros tipos de rubros interdictos por el sistema legal peruano como la prostitución, la delincuencia, la trata de personas o el narcotráfico; sin embargo, las estadísticas mencionan que la presencia de la población venezolana en estos rubros es menor al $1 \%$ pero sí existe, si se da y cada día se viene desarrollando más a espaldas de un Estado ausente.

Blouin \& Freier (2019) sostienen que el proceso de inserción de la población venezolana en el Perú ha transitado por diversas aristas y reglamentaciones que ha hecho inviable la inserción de estos al mercado laboral formal debido a las altas exigencias a lo que concluyen que "como se puede ver, existen una serie de obstáculos a la contratación formal que la vuelve poco atractiva tanto para los empleadores como para las personas migrantes" (p. 177). Originando que gran parte de esta población migre hacia el sector informal, lo cual, vemos en ejemplos tan claros como los repartidores de comida rápida mediante los aplicativos Rappi o Pedidos Ya; según las cifras no oficiales dadas por diversos medios se estima que un $96 \%$ de los repartidores son venezolanos. Por lo cual, concluiremos que la población venezolana en el Perú no tiene derechos laborales, no existe una institución que vele por ellos dentro del mercado laboral y las políticas públicas relacionadas a la empleabilidad no ha considerado dentro de ellas a la población 
venezolana. La remuneración que percibe se encuentra por debajo del mínimo y son explotados en jornadas de más de 8 horas, ocasionando que la calidad de vida de ellos se encuentre en una situación crítica.

Un estudio psicológico elaborado por Figueroa-Quiñones et al., concluye que "la frecuencia de presentar ansiedad/depresión fue mayor en aquellos migrantes venezolanos con grado de instrucción superior universitario en comparación con aquellos con estudios de secundaria. Esta asociación se mantuvo al ajustar por el número de horas laborales" (p. 383).

La precarización laboral en el Perú sería el detonante del proceso de estigmatización a la que fue sometida la población venezolana mostrándola como los parias a los que se les debe de hacer frente, a los que "debemos de combatir porque nos quitan el pan", frases que suelen ser muy escuchadas en la calle, en reuniones familiares, en reuniones sociales, entre otros espacios de socialización.

Ante este panorama una gran parte de la población peruana se sintió desplazada, emergiendo discursos dentro de la población como: "estos venecos han venido a quitarnos el trabajo". O discursos dentro de la política peruana como:"no es posible que vengan extranjeros y nos quiten nuestros puestos de trabajo". 0 discursos nacionalistas que hemos visto durante la presente campaña electoral 2021, quien enarbola esta bandera no era nada menos que Rafael López Aliaga o Daniel Urresti. Existía una indignación dentro de la población, que los medios de comunicación supieron canalizar a través de sus redes a la comunicación, ello, lo continuaremos en el siguiente apartado.

\section{Del halago al insulto: proceso de estigmatización de la comunidad venezolana en el Perú}

En este apartado veremos cómo la población venezolana en el Perú ha sido estigmatizada por parte de la población peruana; emergiendo discursos xenófobos, racistas y nacionalistas. Por ello, en una primera instancia haremos una reflexión teórica del migrante, considerado el "otro", el "forastero" o el "enemigo". Para luego, analizar el proceso de estigmatización a la que ha sido sometida la población venezolana en el Perú tomando como referencia la construcción social del venezolano que se ha dado desde los medios de comunicación. Sin embargo, al ser un dominio muy extenso por cuestiones metodológicas, ontológicas y gnoseológicas nos centraremos en el proceso de estigmatización que se dio desde el diario El Comercio viendo como desde el 2016 al 2021 han variado sus notas y contenidos con respecto a la población venezolana en el Perú. De esta forma, veremos como a través de los medios 
de comunicación, la población peruana no ha hecho más que reforzar dentro de su idiosincrasia aquellos discursos xenófobos, racistas, alienados que existían hacia la población venezolana de manera extraoficial.

Una de las primeras reflexiones hechas sobre el rol y la figura del migrante desde la sociología la podemos encontrar en el escrito de Alfred Schutz, "El forastero. Ensayo de psicología social" del año de 1974. Esta obra tiene como finalidad proponer una teoría interpretativa general acerca de la situación en la que se encuentra un forastero al insertarse e interpretar el esquema social y cultural de un grupo determinado, diferente al suyo, en aras de ser aceptado dentro de este. Por esta razón, el ejemplo más resaltante es la del inmigrante; el análisis presente busca englobar otros casos como la del postulante a un club, el recluta, entre otros ejemplos.

Se excluye de la definición de forastero al visitante, al turista, a los niños porque su proceso como tal es transitorio y eventual. Como punto de partida propone la examinación de la "pauta cultural de la vida grupal" de los individuos, la que es entendida como todas las valoraciones, instituciones, costumbres, leyes y etiquetas que el migrante debe de apropiárselas para poder pertenecer al grupo social. Como señala Schutz (1974) "todo miembro nacido o educado dentro del grupo acepta el esquema estandarizado ya elaborado de la pauta cultural recibida de sus antepasados, como una guía indiscutible en todas las situaciones que se dan normalmente en la vida social" (p. 98).

El conocimiento del ser humano que se desarrolla en la cotidianidad de los individuos no es homogéneo. Al contrario, este es incoherente, solo parcialmente claro e incongruente. No obstante, el sistema de conocimiento que adquieren los miembros del grupo toma la coherencia, claridad y congruencia suficiente para comprender, pensar y razonar la vida social. Por lo cual, resulta importante aquí el lugar de pertenencia social y el de enunciación; puesto que ello marcará y evidenciará las diferentes categorías existentes dentro de los marcos sociales.

El forastero al no compartir este sistema de conocimiento del endogrupo cuestiona todo acerca de este; para él la pauta cultural de dicho grupo no tiene la autoridad suficiente. Razón por la cual, el forastero a lo sumo puede compartir el presente y el futuro en experiencias vividas e inmediatas. Ante esta situación el forastero deja de ser un observador no participante para convertirse en aspirante a un miembro del grupo que se acerca donde la actitud del forastero se basará en la objetividad de este y la dudosa lealtad, "para el forastero, la pauta cultural de su grupo de origen sigue siendo el resultado de una evolución histórica ininterrumpida y un elemento de su biografía personal...es el esquema incuestionado de referencia para su 'concepción relativamente natural del mundo'"' (Schutz, 1974, 
p. 100). Es a partir de estos sucesos donde el migrante entra en conflicto al tener que adquirir una nueva pauta cultural diferente a la suya. De igual forma, las personas pertenecientes al endogrupo entran en conflicto al ver al migrante que no se adecua a sus tradiciones.

Por lo cual, Schutz (1974) concluiría que "la adaptación del recién llegado al endogrupo que al principio le parecía extraño y desconocido, es un proceso continuo de indagación en la pauta cultural del grupo abordado" (p. 107). Por lo que podemos deducir que quienes no se adecuen a la pauta cultural del grupo queda relegado y es estigmatizado por el endogrupo. Situación que pasa la población venezolana en el Perú:

Se trata de un fenómeno que despertó, en un inicio, una exótica forma de visualizar a los recién llegados, para convertirse, poco a poco, en una percepción discriminatoria y xenófoba, llegando a los propios medios de comunicación que no dejaron de apelar a la incertidumbre y al miedo de la presencia de tal cantidad de migrantes (Loayza \& Ticona, 2021, p. 74).

Por tal motivo, examinaremos las noticias publicadas en el diario El Comercio sosteniendo la tesis que los medios de comunicación fueron un factor clave en la construcción social del migrante venezolano en el Perú, pasando del "refugiado venezolano que huye de una crisis política, social y económica desatada por el gobierno de Nicolás Maduro" al "venezolano frustra robo" para terminar en un "Migrantes venezolanos acaparan el sector informal de trabajo ocasionando miles de desempleos para la población peruana". En el año 2016, El Comercio publicó 4 noticias sobre la población venezolana en el Perú. De los cuales, todas muestran al venezolano como víctima de un modelo económico perverso y del chavismo que están siendo refugiados por el Perú. De igual forma, presentan noticias de sobre como los venezolanos refugiados (término que se usó en aquellos años pero que ahora ha cambiado significativamente evidenciando que la percepción que se tenía hacia ellos se ha negativizado) veían la situación política, social y económica en su país.

Para el 2017, este mismo diario publicó 14 noticias sobre la población venezolana en el Perú. Una de las conclusiones a la que podemos llegar, es la construcción social del migrante venezolano como un emprendedor que vende de manera ambulante sus arepas, tizanas y bombitas, aquel al que la población peruana debía de ayudar y tener consideración. Asimismo, se evidencia que otro aspecto que resaltaba por aquellos años este diario es mostrar al Perú como un país solidario y que ello, lo dicen los diversos organismos internacionales (tomando como referencia aquel 
discurso del entonces presidente Pedro Pablo Kuczynski en la OEA cuando abre las fronteras a la población migrante venezolana dándoles en trato de refugiados). Así, el diario en mención comenzó a desarrollar una línea de noticias del venezolano víctima del chavismo y un país entre la crisis y la catástrofe.

Ello no hizo más que gran parte de la población vea en la población migrante a un amigo al que se le debe de tender la mano. Siguiendo esta misma línea, se presentaron entrevistas a venezolanos en el Perú que se oponían al régimen de Maduro. De manera paralela, publicaron noticias de prevención al migrante venezolano de la estafa y la explotación que podría hacerle el peruano o presentaban casos de venezolanos que se habían inmolado ayudando a una peruana, dando su vida por ella.

La percepción de la población en el 2017 hacía el venezolano, siguiendo la línea de los medios de comunicación era positiva, es más, muchos peruanos veían de manera positiva la presencia de los venezolanos porque subían los estándares raciales, de hecho, ese año surgió la cosificación hacia la mujer venezolana por parte del hombre, la cual comenzará a ser vista como un trofeo para vanagloriarse ante los demás y discursos racistas, tales como: "con los venezolanos acá, se mejorará la raza" o "así estos cholos serán desplazados". Es en este año, donde las empresas comienzan a contratar a personas venezolanas para trabajar en áreas de atención al cliente. Un reportaje de este año hecho por Panorama revela como en Gamarra comenzaron a abundar anuncios de trabajo para señoritas venezolanas "de buena presencia" para modelar ropa.

En el 2018, si bien no existe un ruptura ni estigmatización a la población venezolana, las noticias variaron considerablemente con respecto al año anterior. En este año, El Comercio presentó un total de 48 noticias de las cuales 7 eran sobre venezolanos que delinquen, mas seguían con la línea de construcción del venezolano amigo que te ayuda con noticias como "ciudadano venezolano frustra un asalto", quizás, un héroe popular migrante es el venezolano.

Otra noticia muy resaltante de este año es una en la que presentaban a los diversos migrantes venezolanos que han venido al país, su historia de superación, sus aspiraciones; en otras palabras, le daban un rostro al ciudadano venezolano refugiado (aún el término migrante no era muy usado). Al darle un rostro, se intentaba llegar más a la población, concientizarla y comprometerla. Apelando a la solidaridad y sacando notas sobre asesoría legal para la población venezolana en el Perú. Pero a mediados de año, las noticias cambian de tonalidad, ya no se hablaba de refugiados, sino de un éxodo sin precedentes de migrantes venezolanos que venían al Perú, mostrando las diversas historias dramáticas, pero también mostrando 
el peligro que puede resultar el migrante venezolano si no existen parámetros en migraciones.

Por esta razón, comienzan a salir artículos en este diario que hablaban sobre el impacto que los migrantes venezolanos podrían tener en la economía nacional, la necesidad de tener una política migratoria o cuestionando a la población migrante que venía al Perú. Se comienzan a abordar temas más ligados al proceso migratorio, y el 25 de octubre se publica una nota en donde se alababa al Perú como el país más solidario con los venezolanos.

De manera paralela, desde setiembre este diario comenzó a publicar artículos en contra de la xenofobia y sobre la importancia de la solidaridad. Puesto que, dentro de la población se estaban comenzado a desarrollar discursos racistas hacia los venezolanos por la atención que le estaban dando los medios, el Estado y la inserción de estos al sistema laboral, sistema de salud y al sistema educativo como parte de una estrategia de integración nacional que adoptó el país creando un protocolo para la población venezolana. En octubre, se da la primera alerta de xenofobia en el Perú. Para ese mismo año se anuncia un apoyo económico de EE. UU. de unos 4.1 millones de dólares para apoyar a la población venezolana en el Perú; pero para finales de año. Este mismo año del 26 de noviembre al 31 de diciembre se realizó "Encuesta dirigida a la población venezolana que reside en el País" (EMPOVE 2018) por el INEI, el objetivo principal de esta fue de "brindar información sobre las condiciones de vida de la población venezolana residente en el país; caracterizando los aspectos demográficos, sociales, situación migratoria, discriminación, violencia. Así como, las características de la vivienda, acceso a servicios básicos y equipamiento del hogar" (EMPOVE, 2018, p. 17). Una primera aproximación estatal al fenómeno de la migración venezolana en el Perú. No obstante, la difusión de esta y de sus resultados ha sido inexistente por parte de los medios de comunicación.

El 2019 será un año de transición, de redefinición y de estigmatización al migrante venezolano que se hará visible a partir del segundo semestre. En este año se publicaron alrededor de 97 noticias sobre la población venezolana en el Perú, de las cuales 47 presentan al venezolano de manera negativa y 6 tratan sobre la situación política de Venezuela, la disputa de poderes entre Guaidó y Maduro por la presidencia del país. Aquel año comenzó con el titular:"Venezolanos entregaron a dos de sus compatriotas acusados de robar" (Redacción EC, 2019). Como hemos de ver, los primeros meses del 2019 El Comercio seguía la misma línea del 2018, se presentaba lo positivo de la migración venezolana y la construcción social del venezolano de igual forma era positiva. Los titulares siguientes a esta noticia estaban relacionados a las elecciones venezolanas del 2019, en donde Nicolás Maduro 
logra su reelección. Situación que creó una época de conflicto donde la oposición en venezolana convoca manifestaciones en todo el país.

La repercusión de este suceso tuvo eco en la iglesia católica, la conferencia episcopal venezolana considera ilegítimo el nuevo mandato de Nicolás Maduro (Redacción EC, 2019). Asimismo, un reporte hecho por la BBC (2019) titulado"Crisis en Venezuela: qué dice la carta del papa Francisco a Nicolás Maduro y por qué se ha interpretado como un revés para el mandatario"y publicado en su página web, señala que el papa Francisco remitió una misiva al mandatario venezolano donde criticó su régimen y le solicitó "evitar cualquier derramamiento de sangre" por la represión que hacía su régimen al intentarse aferrarse al poder.

Es así como El Comercio comienza a abordar esta situación desde la percepción de los venezolanos en el Perú haciendo una recopilación de multimedios, testimonios e historias de migrantes venezolanos víctimas del régimen chavista.

Un hecho relevante a mencionar es la manifestación que se dio en enero al frente de la embajada de Venezuela contra la reelección de Nicolás Maduro. Este mismo diario siguió aquella línea política de presentar las protestas y el levantamiento de los militares en contra de Maduro, creando una expectativa muy interesante en los lectores y abonados de su diario.

El diario El Comercio en su página web, el 1 de febrero publicó la nota informativa: "Allegados de Guaidó reconocen al Perú como uno de los países más solidarios" (2019). En un contexto político internacional del reconocimiento de varios países, como EE. UU., Brasil, la Unión Europea, Perú, entre otros más, a Guaidó como nuevo presidente interino de Venezuela, luego de su proclamación hecha por la Asamblea Nacional de Venezuela el 23 de enero del 2019.

Dentro del panorama nacional, el 26 de febrero se publica la siguiente nota en este diario: "Julio Velarde: Migración venezolana impactó en los salarios informales". Noticia que analizaremos desde dos perspectivas, la primea es quien la enuncia y la segunda el contenido de esta. Para responder a la primera cuestión hemos de ver que Julio Velarde es desde el 2006 presidente del Banco de Reserva del Perú, designado inicialmente por Alan García y ratificado por los gobiernos siguientes. El contenido de la noticia toca un tema que desde el 2000 estaba en discusión que es: el sistema laboral en el Perú, el cual estará marcado por tres características: i) elevadas tasas de desempleo; ii) precarización laboral; iii) la no atención por parte de los gobiernos de turno en crear políticas públicas en pro de mejorar el sector laboral en el país.

Ello ocasionó, la fuga de mano de obra al sector informal de trabajo que según las últimas cifras presentadas por el diario La República representa a un total de 
$71,1 \%$ de trabajadores. Es decir, que solo un $29,9 \%$ de trabajadores sí cuentan con derechos laborales. Situación que de por sí en la población peruana creaba un malestar, este se acrecentó y se centró en la población venezolana. Una siguiente nota escrita el 22 de marzo por Katherine Maza, siguiendo la misma línea, lleva como titular: "Alonso Segura: Migración venezolana podría tener incidencia sobre la pobreza en el Perú". Posterior a estas primeras aproximaciones sobre la migración venezolana en el Perú desde los ámbitos económicos y laborales y sus consecuencias en el país, se publica una nota en la que se cuestionaba al alcalde de Huancayo por sus declaraciones xenofóbicas.

Pero, para el mes de abril se publica un informe de la Cancillería del Perú avizorando y advirtiendo a la población sobre el impacto de la migración venezolana en el mercado laboral, la disminución de las postas de trabajo, la disminución de los sueldos y el giro de varias empresas en comenzar a contratar a migrantes venezolanos desplazando a la población peruana. Esto hizo que el contenido de las siguientes notas fuese cambiando, ya no se presentaba al refugiado venezolano en el Perú, sino que se comenzó a hablar del migrante venezolano que trabaja en el sector informal, como se evidencia en el siguiente titular:"Denuncian que peruanos y venezolanos atienden en policlínicos de Lima sin títulos profesionales".

Por otro lado, ante las crecientes olas de xenofobia y discriminación que se comenzaban a manifestar en la sociedad peruana UNICEF, la Embajada de Canadá y Migraciones lanzan una campaña para el buen recibimiento de migrantes venezolanos con el \#RecíbelosConAmor. No obstante, para abril de ese mismo año el panorama da un giro de $180^{\circ}$, las noticias presentadas por el diario en mención hacen alusión a un desborde popular dentro de la capacidad laboral del Perú debido al auge de venezolanos.

De allí, en adelante, los medios de comunicación comienzan a mostrar a los migrantes venezolanos desde otras perspectivas que, si bien son variados, se presenta a la población venezolana de una forma menos simpática para los lectores, titulares como: "venezolanos asaltan tiendas" o "venezolano asesina a joven para robarle sus pertenencias" comienzan a tener mayor reproducción y acaparamiento por los medios de comunicación.

Ello evidencia una ruptura con la construcción social anterior del migrante venezolano refugiado al que se le tenía que ayudar a un venezolano que ha venido a delinquir en nuestro país, estas noticias comienzan a reforzar y dar sustentos a los discursos xenofóbicos que iban surgiendo dentro de la sociedad peruana y consolida a partir de ello, el proceso de estigmatización de la población venezolana en el país, desde una lectura negativa a su presencia. Las noticias que continuaron desde 
abril a fin de año giraban más en torno a venezolanos dentro de la delincuencia, venezolanas dentro de redes de prostitución, venezolanos en redes de estafa, venezolanos dentro del sicariato, etc.

A finales de ese mes, El Comercio publica la siguiente noticia: "INPE: Población venezolana en prisión se sextuplicó en solo un año" (Redacción EC, 2019), lo que marcó seriamente la nueva construcción social que se le hacía al venezolano al no adscribirse a la pauta cultural peruana. Asimismo, las noticias sobre el impacto de los venezolanos en el sector laboral no hacían más que acrecentar la ruptura de una población peruana golpeada por el desempleo con los migrantes venezolanos que se iban insertando al sector laboral.

Dentro de este panorama, desborde popular, sector laboral en colapso y discursos xenofóbicos emergentes, Vizcarra anuncia el proyecto "Plan Migratorio 2019" en donde a partir del 15 de junio del 2019 anuncia que se solicitará visa humanitaria a los venezolanos. Esta disposición causo polémica a nivel internacional debido a que algunos mandatarios como Nicolás Maduro, consideraban este acto como un atentado a los derechos humanos y a los refugiados. Acto que el mandatario venezolano respondió de igual forma.

Dentro del discurso de Martin Vizcarra sobresalió la siguiente frase: "Después de abrirles los brazos, es lógico que pidamos visa". Las noticias siguientes en este diario, seguían dentro de la misma línea trazada desde abril y giraban en torno a temáticas como: venezolano acosa a jovencitas, banda de venezolanos es desarticulada por la policía nacional, venezolanos que viven en la pobreza, venezolano sicario asesina a jóvenes en el distrito $x$, venezolanos ingresan de manera ilegal al país, etc. Situación que agravó la convivencia social entre la población peruana con la población venezolana, los discursos xenofóbicos se trasladan a la política peruana a través de diversos congresistas quienes optaron por enarbolar la bandera de un discurso nacionalista proteccionista. Fue tanto el rechazo que generó una gran parte de la población peruana a los migrantes venezolanos (véase el último informe del INEI) que Nicolás Maduro y Juan Guaidó exigieron y solicitaron a Martín Vizcarra que tomase medidas correctivas al incremento de la xenofobia y es así como el Perú, luego de haber sido catalogado como el país más solidario se convirtió a los ojos internacionales como el país más xenofóbico.

Para el año 2020, El Comercio publicó 61 notas relacionadas a la población venezolana en el Perú; recordemos que este año se dio inicio al confinamiento social para evitar la propagación de la CovID-19 pero las repercusiones sociales y económicas golpearon a gran parte de la población como hemos mencionado en apartados anteriores. De las 61 noticias, 37 hablan sobre estafa, asesinatos, 
violaciones, informalidad y prostitución ocasionados por los venezolanos y las venezolanas en el Perú transgrediendo las normas sociales. En el 2021, hasta el 26 de abril hemos de ver que este diario ha publicado un total de 21 noticias sobre la población venezolana en el Perú en donde 10 abordan las temáticas anteriores sobre el estigma del venezolano.

Por lo cual, concluiremos que los medios de comunicación han tenido un rol activo en el proceso de estigmatización de la población venezolana. En los primeros años, 2016 al 2018, se presentaba al migrante venezolano como un refugiado político de un país en crisis y desborde. Ante este panorama, se le dio un rostro al migrante venezolano y se inició una línea de noticias con la finalidad de concientizar a la población peruana y a su vez, el tender la mano a los refugiados venezolanos. Sin embargo, para el 2019, con los pronunciamientos de Julio Velarde, Mercedes Aráoz, Alonso Segura, entre otros, quienes señalaron como negativo la presencia de los migrantes venezolanos para la economía peruana y para el sistema laboral, los lineamientos de noticias cambiaron radicalmente. Se dejó de presentar al "venezolano refugiado emprendedor" para presentar al ahora "venezolano inmigrante delincuente".

Ello influenció radicalmente en la población peruana ocasionando que a partir del 2019 existiese un incremento exponencial de discursos racistas y xenofóbicos hacia la población venezolana. Asimismo, podemos ver dos iniciativas que surgieron para evitar el colapso social. La primera fue elaborada por la policía nacional del Perú con la creación de una brigada especial contra migrantes venezolanos. Como señala un informe hecho por la BBC (2020):

"Esos venezolanos y venezolanas que han ingresado a delinquir tienen solo dos caminos: irse del país o la cárcel" - Con estas palabras, el ministro del Interior de Perú, Carlos Morán, anunció la "brigada especial contra migración delictiva" en la policía peruana. Eso, a pesar de que los delitos cometidos por extranjeros en Perú suman menos del 2\%, según cifras de 2019 del Ministerio del Interior difundidas por medios peruanos.

De hecho, la designación de esta brigada no solo generó un incremento considerable de xenofobia en el país, sino que provoco el reforzamiento al estigma que ya los medios de comunicación habían hecho del migrante venezolano a partir del 2019. Un aspecto relevante por mencionar es que estadísticamente los delitos cometidos en el país de extranjeros con la justa suma el $2 \%$ del total, los medios de comunicación han construido la percepción que esta es mucho mayor (como hemos de ver, en todas las notas desde el 2019 las noticias negativas en torno a la 
población venezolana han representado un poco más del 50\%) generando que la población peruana consideré como amenaza a la población venezolana en el país.

Por otro lado, debido a este panorama la UNHRCR-ACNUR lanzó la campaña \#TuCausaEsMiCausa con la finalidad de frenar la xenofobia, discriminación y racismo hacia la población venezolana y poblaciones vulnerables en el país.

\section{Pandemia, olvido y silencio: situación de la población venezolana en el Perú}

La población venezolana en el Perú durante la crisis sanitaria ha sido afectada intersecionalmente por diversos factores, una población bordeando la informalidad, en índices de pobreza, estigmatizada y sin accesos a los servicios básicos. Una población venezolana que viene siendo invisibilizada por los medios de comunicación desde que se inició la pandemia. De la Cruz (2020) señala que:

La falta de calidad en el empleo, la flexibilización del mercado laboral y la precarización de las condiciones laborales son, hoy en día, causas que inciden de manera directa en las tasas de pobreza. Sus efectos tienen mayor repercusión en la calidad de vida en aquellas personas o grupos que están en una situación de mayor fragilidad en ese contexto: niños que viven en hogares con bajas tasas de trabajo; personas con discapacidad, que son las que mayor privación material severa padecen (p.329).

Siguiendo el planteamiento hecho por esta autora, podemos entonces afirmar que la población venezolana desde una perspectiva intersecional es una de las poblaciones más afectadas por todas estas variables, convirtiéndola en la actualidad como una de las poblaciones más vulnerables frente a la pandemia originada por la CovID - 19 y frente a una crisis sanitaria ocasionada por el mal manejo de políticas públicas desde el aparato estatal.

Esta situación, es corroborado por el último informe del Banco Mundial elaborado por María Dávalos y titulado:"Migrantes y refugiados venezolanos en el Perú: el impacto de la crisis de la CoVID -19". Por tal razón, presentaremos y examinaremos cada impacto que ha podido encontrar la autora en relación con la situación de la población venezolana en el Perú.

En correlación con el marcado laboral señala que "los migrantes y refugiados venezolanos se encuentran en empleos más vulnerables, debido a la alta informalidad y su concentración de pequeñas empresas" (Dávalos, 2020, p. 1). Esta fragilidad en las condiciones de trabajo ha hecho que los migrantes venezolanos acepten 
condiciones laborales sin acceder a sus derechos fundamentales como vacaciones, CTS., seguridad social, etc. Asimismo, muchos venezolanos han optado por el trabajo independiente como ambulantes en las calles más concurridas de Lima metropolitana. Sin embargo, habremos de mencionar que las migrantes venezolanas desde la perspectiva de la interseccionalidad son mucho más afectadas que el migrante venezolano debido a su condición como mujer; muchas de ellas o han sido víctimas de acoso o se han visto obligadas a prostituirse para lograr conseguir recursos para sobrevivir. Por ello, hemos elaborado un diagnóstico basándonos en dos páginas de internet " $x$ " $y$ " $z$ " en donde se ofrecen servicios sexuales bajo la denominación de kinesiólogas. En cada página hemos muestreado 100 anuncios de kinesiólogas en Lima metropolitana. En la página " $x$ " las venezolanas representaban un $73 \%$ de los anuncios, las edades iban desde los 18 años hasta los 24 años, el $60 \%$ de ellas se centraban en el distrito de Los Olivos, específicamente por Mega Plaza. Mientras que en la página " $z$ " las venezolanas representaban un $64 \%$ de los anuncios, las edades encontradas estaban desde los 18 años hasta los 31 años, de los cuales un $50 \%$ se encontraban en Los Olivos, un 25\% en San Borja, un 11\% en Surco, 9\% en Miraflores y un $5 \%$ en otros distritos.

Por otro lado, hemos de ver que "los migrantes y refugiados venezolanos están representados en los sectores más afectados" (Dávalos, 2020, p. 1). Los sectores laborales que habían absorbido a la población venezolana eran: hotelería, gastronomía, comercio, transporte y construcción. Estos han sido los sectores económicos más golpeados por la pandemia, ocasionando que millones en el Perú perdiesen su trabajo, sumiendo a la pobreza millones de hogares en el Perú.

Dávalos detalla que"Ios migrantes venezolanos y refugiados están más concentrados en las áreas urbanas, las más afectadas por el shock" (2020, p. 1). Tomando ciertos aspectos estructurales del país, podemos concluir que no ha existido una política descentralizadora de los servicios básicos para la población en general. Ello ha ocasionado que toda la población se haya aglomerado en Lima y otras ciudades donde el proceso de urbanización se ha dado con mayor rapidez que en otras. La sobrepoblación de estos espacios ha hecho que el costo de vida se incremente considerablemente, ello ocasiona la marginalización de una gran parte de la población quienes no tienen los recursos necesarios para poder subsistir obligándolos a tener que ingeniarse formas para poder incrementar sus ingresos económicos.

Asimismo, Dávalos (2020) señala que "los migrantes y refugiados venezolanos ganan menos que los peruanos y tiene niveles de pobreza más altos" (p. 1). Debido a como la masa trabajadora venezolana en el Perú ha sido absorbida por el sector informal, en donde los empleadores no reconocen derechos laborales ni igualdades 
salariales entre la población venezolana con la población peruana. Por este motivo, ante la no responsabilidad jurídica estas empresas siguen contratando mano de obra venezolana porque le sale mucho más cómoda. Todo ello es posible, debido a que no existe una política regularizadora y ningún gobierno ha tomado ello en consideración.

En relación con el acceso a servicios Dávalos menciona que "los migrantes y refugiados venezolanos tiene menor acceso a servicios de salud" (2020, p. 1). Ello se debe a la inexistencia de una política integradora dentro del sistema de salud, como también a la no renovación del sistema de salud. Esta es la razón principal por la cual, la población venezolana en el Perú no puede acceder a un tratamiento integral contra la COVID-19, pasando por la penuria de un servicio burocrático, ineficiente y, hasta cierto punto, informal generando una disminución al porcentaje de sobrevivencia frente a esta enfermedad. De esta forma, se le es negado el acceso a un derecho humano universal que es el del acceso a un sistema de salud (sumándole otros sistemas a los cuales la población venezolana es marginada como el de educación, trabajo, etc.).

Posteriormente Dávalos (2020) subraya que "los migrantes y refugiados venezolanos tiene un acceso muy limitado a programas sociales y de empleo (p. 2). Esto se debe principalmente a que la población venezolana no está incluida dentro del Sistema de Focalización de Hogares (SISFOH). Al no ser considerada dentro de esta, esta población queda en vulnerabilidad frente a una crisis sin poder acceder a los servicios básicos de sobrevivencia. En un reportaje de América Televisión sobre"Ios ambulantes de la pandemia" al entrevistar a un migrante venezolano, el sostuvo la siguiente frase: "prefiero morir de CovID-19 que de hambre".

Finalmente, Dávalos (2020) sostiene que "estas vulnerabilidades se exacerban ante las condiciones de vivienda de esta población" (p. 2). No toda la población venezolana en el Perú puede acceder a una vivienda propia. Según datos del Banco Mundial, solo un $95 \%$ de ellos viven en una vivienda rentada.

\section{Conclusiones}

La población venezolana en el Perú es la población extranjera más grande que representa a casi 1 millón 200 mil personas. Sin embargo, es la población más vulnerable en la actualidad frente a la crisis sanitaria generada por la covid-19. Esto se debe a que estructuralmente el Perú carecía de una política pública migratoria que pudiese orientar el proceso. De igual manera, la pandemia no ha hecho más 
que visibilizar un sistema de salud defectuoso, sistema de educación exclusivo y un sistema laboral precarizado.

Los migrantes venezolanos mueren a causa de la COVID -19 en el país, pero ni los medios de comunicación ni el Estado ni los gobiernos han intentado visibilizar esta problemática social, negándoles sus derechos básicos de salud, educación y una vida digna. En ese sentido, no han existido políticas públicas desde el Estado para: i) ordenar a la población migrante; ii) censar a la población migrante; iii) empadronar a la población migrante dentro de los servicios básicos. Al no existir una política migratoria, la población migrante en el país fue absorbida por el sector informal de trabajo. Por otro lado, al no existir un verdadero censo de la población migrante en el país, las estadísticas se han basado en aproximaciones a cifras del Banco Mundial y Migraciones, pero no se sabe a ciencia exacta cuál es el número real de migrantes venezolanos en el país. Al no ser empadronada esta población, casi un $80 \%$ no puede acceder a los servicios básicos y sus condiciones de vida han empeorado considerablemente desde el inicio del confinamiento social.

Las primeras olas de migrantes venezolanos pudieron ser absorbidas por el sector laboral formal de trabajo; sin embargo, ante el auge de migrantes venezolanos en el país ocasionó una sobrepoblación de mano de obra en el mercado. Ello hizo, que muchos venezolanos fuesen absorbidos por el sector informal de trabajo, aceptando condiciones deplorables en jornadas mayores a las ocho horas con sueldos bajo el salario mínimo vital. Un ejemplo de la precarización laboral en el Perú a la que la población venezolana ha sido sometida la encontramos en las venezolanas migrantes de 18 a 24 años que en diversas páginas de kinesiólogas ofrecen servicios sexuales a cambio de una remuneración que va desde los $100 \mathrm{a}$ 250 soles.

Por otro lado, los medios de comunicación fueron un factor clave en la estigmatización de la población venezolana en el Perú como algo negativo. Razón por la cual, desde el 2019 en el Perú se han incrementado discursos xenofóbicos en contra de la población venezolana. 


\section{Referencias}

Banco Mundial (2020). Infografía: Migrantes y refugiados venezolanos en el Perú: El impacto de la crisis de la Covid-19 (\#Coronavirus). enero 23, 2021, de Banco Mundial Sitio web: https://www.bancomundial.org/es/news/infographic/2020/07/22/ infografiamigrantes-y-refugiados-venezolanos-en-el-peru-el-impacto-de-la-crisis-de-lacovid-19-coronavirus

BBC Mundo (2019). Juan Guaidó se autoproclama "presidente encargado": quépaíses reconocen al presidente de la AN y cuáles se mantienen con Nicolás Maduro. 27 abril, 2021, de BBC: https://www.bbc.com/mundo/noticias-internacio $\neg$ nal-46982431

BBC News Mundo (2019). Crisis en Venezuela: el gobierno de Perú pedirá visa a los inmigrantes venezolanos. Abril 27, 2021, de BBC: https://www.bbc.com/ mundo/ noticias-48551096

BBC News Mundo (2020). Mayo 2, 2021. Migración venezolana en Perú: la polémica por la creación de una brigada policial contra la "inmigración delictiva", de BBC: https:// www.bbc.com/mundo/noticias-america-latina-51215514

Bedoya, D. (2019). INPE: Población venezolana en prisión se sextuplicó n solo un año. mayo 1, 2021, de El Comercio: https://elcomercio.pe/lima/sucesos/inpe-poblaาcion-venezolana-prision-sextuplico-ano-infografia-noticia-ecpm-631252-noticia/

Blouin, C. (2019). Después de la llegada: realidades de la migración venezolana.

Blouin, C., \& Freier, L. F. (2019). Población venezolana en Lima: entre la regularización y la precariedad. L. Gandini, F. Lozano y V. Prieto (coords.) Crisis y migración de población venezolana. Entre la desprotección y la seguridad jurídica en Latinoamérica. México: UNAM.

Condori, M., Reyna, G., Villavicencio, A., Párraga, C., y Vilcapoma, D. (2020). Éxodo venezolano, inserción laboral y discriminación social en la ciudad de Huancayo, Perú. Revista ESPACIOS. ISSN, 798, 1015.

D’Olmo, G. (2019). Crisis en Venezuela: qué dice la carta del papa Francisco a Nicolás Maduro y por qué se ha interpretado como un revés para el mandatario. Abril 27, 2021, de BBC: https://www.bbc.com/mundo/noticias-america-latina-47233637

Dávalos, M. (2020). Migrantes y refugiados venezolanos en el Peru: El impacto de la crisis de la covid-19 (No. 149108, pp. 1-3). The World Bank.

De Cruz-Ayuso, C. (2020). Repensar la política de lucha contra la pobreza y la exclusión desde el prisma de la interseccionalidad. Cuadernos salmantinos de filosofía, 47, 327-349.

Echevarria, E. (2018). Los derechos laborales y la migración venezolana en el Perú.

Economía LR. (2020). El 71,1 \% de trabajadores son informales en el Perú. Mayo 2, de La República: https://larepublica.pe/economia/2020/04/02/71-de-traba-jadoresson-informales-en-el-peru/?ref=Ire

Hernández, A., Vargas, R., Rojas, C., y Bendezu, G. (2020). Factores asociados a la no utilización de servicios de salud en inmigrantes venezolanos en Perú. Revista Peruana de Medicina Experimental y Salud Pública, 36, 583-591. 
Figueroa, J., Cjuno, J., Ipanaqué, J., Ipanaque, M., y Taype, A. (2019). Calidad de vida de migrantes venezolanos en dos ciudades del norte del Perú. Revista Peruana de Medicina Experimental y Salud Publica, 36, 383-391.

INEI (2018). Condiciones de vida de la población venezolana que reside en Perú. INEI, Lima, Perú. Koechlin, J., Vega, E., y Solórzano, X. (2018). Migración venezolana al Perú: proyectos migratorios y respuesta del Estado. 31.

Loayza, J., y Ticona, R. (2021). Miedo, desarraigo y proyectos familiares en la población inmigrante venezolana en el Perú. Pluriversidad, (6), pp. 73 - 84.

Maza, K. (2019). Alonso Segura: Migración venezolana podría tener incidencia sobre pobreza en el Perú. mayo 1, 2021, de El Comercio: https://elcomercio.pe/ economia/ peru/alonso-segura-migracion-venezolana-incidencia-pobreza-peru-noticia619050-noticia/

Mendoza, W., \& Miranda, J. J. (2019). La inmigración venezolana en el Perú: desafíos y oportunidades desde la perspectiva de la salud. Revista Peruana de Medicina Experimental y Salud Publica, 36(3), pp. 497-503.

MTPE. (2012). Política Nacional del Empleo. Lima: MTPE.

Parent, N. (2017). La protección se queda corta: el nuevo programa de migración de Perú para los venezolanos.

Portal Turismo (2021). Ahora Perú señala que más de 70,000 restaurantes han cerrado durante la pandemia. Enero 24, 2021, de Cámara Nacional de Turismo del Perú: https:// portaldeturismo.pe/noticia/ahora-peru-senala-que-mas-de-70000-restauranteshan-cerrado-durante-la-pandemia/

Ramirez, J., Linarez, Y. y Useche, E. (2019). Geo-políticas migratorias, inserción laboral y xenofobia: migrantes venezolanos en Ecuador. En Cécile Blouin, Después de la Llegada. Realidades de la migración venezolana. Lima: Themis-PUCP.

Redacción EC. (2019). Chosica: venezolanos entregaron a dos de sus compatriotas acusados de robar. Abril 27, de El Comercio: https://elcomercio.pe/lima/policiales/ venezolanos-entregaron-dos-compatriotas-acusados-robar-chosica-noticia594797-noticia/

Redacción EC. (2019). Huancayo: abren investigación contra alcalde por discriminación a venezolanos. mayo 1, 2021, de El Comercio: https://elcomercio.pe/ peru/junin/ huancayo-abren-investigacion-alcalde-discriminacion-venezolanos-noticia622368-noticia/

Redacción EC. (2019). Iglesia de Venezuela considera ilegítimo el nuevo mandato de Nicolás Maduro. Abril 27, 2021, de ElComercio: https://elcomercio.pe/mundo/ actualidad/ venezuela-iglesia-considera-ilegitimo-nuevo-mandato-nicolas-maduro-noticianndc-595806-noticia/

Redacción EC. (2019). Julio Velarde: Migración venezolana impacta en salarios informales. Abril 27, 2021, de ElComercio: https://elcomercio.pe/economia/peru/julio-velardemigracion-venezolana-causa-alza-salarios-informales-noticia- 611424-noticia/

Redacción EC. (2019). Militares de la GNB se sublevan contra Maduro en cuartel de Caracas |VIDEOS. Abril 27, 2021, de ElComercio: https://elcomercio.pe/mundo/venezuela/ cotiza-venezuela-comando-guardia-nacional-bolivariana-gnb-subleva-nicolasmaduro-noticia-599580-noticia/ 
Redacción EC. (2019). Campaña promueve buen recibimiento de niños y adolescentes venezolanos. mayo 1, 2021, de El Comercio: https://elcomercio.pe/ peru/campanapromueve-buen-recibimiento-peruanos-ninos-adolescentes-venezolanos-noticia630985-noticia/

Redacción EC. (2019). Oposición convoca manifestaciones contra nuevo mandato de Nicolás Maduro. Abril 27, 2021, de El Comercio: https:/elcomercio.pe/mundo/venezuela/ venezuela-oposicion-convoca-manifestaciones-nuevo-mandato-nicolas-maduronoticia-595839-noticia/

Redacción EC. (2019). Vizcarra sobre venezolanos: “Después de abrirles los brazos, es lógico que pidamos visa". mayo 1, 2021, de El Comercio: https:/elcomercio.pe/ peru/ piura/martin-vizcarra-venezolanos-despues-abrirles-brazos-logico-pidamos-visanoticia-ecpm-645037-noticia/

RFI (2019). Perú solicitará pasaporte y visa humanitaria a venezolanos. mayo 1, 2021, de France 24: https://www.france24.com/es/20190607-peru-pasaporte-visahumanitaria-venezolanos

Redacción EC (2021). La pandemia no cesa: Perú supera los 153.000 muertos por la covid -19, según el SINADEF. Mayo 2, 2021, de El Comercio: https://elcomercio. pe/lima/ sucesos/la-pandemia-no-cesa-peru-supera-los-153000-muertos-por-covid-19-segun-sinadef-coronavirus-noticia/

Rojas, C., y Monterroso, C. (2019). Perfil de competencias profesionales de la Población Inmigrante Venezolana al Perú 2017-2018. Revista Arbitrada Interdisciplinaria Koinonía, 4(8), pp. 128-147.

Tenorio, D. (2020). El empleo informal en el Perú: Una breve caracterización 2007-2018. Pensamiento Crítico, 25(1), pp. 51-75.

Schutz, A. (1974). Estudios sobre teoría social. Buenos Aires: Amorrortu. 